\title{
The effect of ICP monitoring in severe traumatic brain injury: a propensity score-weighted and adjusted regression approach
}

\author{
Pål Rønning, MD, PhD, , Eirik Helseth, MD, PhD, ${ }^{1,2}$ Nils-Oddvar Skaga, MD, PhD, ,,6 \\ Knut Stavem, MD, PhD, ${ }^{2,4,5}$ and Iver A. Langmoen, MD, PhD1,2 \begin{abstract}
${ }^{2}$ The Faculty of Medicine, University of Oslo; and ${ }^{4}$ Department of Pulmonary Medicine and ${ }^{5} \mathrm{\varnothing KH}$, Research Centre, Akershus University Hospital, Lørenskog, Norway
\end{abstract} \\ Departments of ${ }^{1}$ Neurosurgery and ${ }^{3}$ Anesthesiology, and ${ }^{6}$ Oslo University Hospital Trauma Registry, Oslo University Hospital;
}

OBJECTIVE The use of intracranial pressure (ICP) monitoring has been postulated to be beneficial in patients with severe traumatic brain injury (TBI), although studies investigating this hypothesis have reported conflicting results. The objective of this study was to evaluate the effect of inserting an ICP monitor on survival in patients with severe TBI.

METHODS The Oslo University Hospital trauma registry was searched for the records of all patients admitted between January 1, 2002, and December 31, 2013, who fulfilled the Brain Trauma Foundation criteria for intracranial hypertension and who survived at least 24 hours after admission. The impact of ICP monitoring was investigated using both a logistic regression model and a multiple imputed, propensity score-weighted logistic regression analysis.

RESULTS The study involved 1327 patients, in which 757 patients had an ICP monitor implanted. The use of ICP monitors significantly increased in the study period $(p<0.01$ ). The 30 -day overall mortality was $24.3 \%$ ( 322 patients), divided into $35.1 \%$ (200 patients, 95\% confidence interval [Cl] 31.3\%-39.1\%) in the group without an ICP monitor and 16.1\% (122 patients, 95\% Cl 13.6\%-18.9\%) in the group with an ICP monitor. The impact of ICP monitors on 30-day mortality was found to be beneficial both in the complete case analysis logistic regression model (odds ratio [OR] 0.23, 95\% Cl $0.16-0.33)$ and in the adjusted, aggregated, propensity score-weighted imputed data sets (OR $0.22,95 \% \mathrm{Cl} 0.15-0.35$; both $p<0.001)$. The sensitivity analysis indicated that the findings are robust to unmeasured confounders.

CONCLUSIONS The authors found that the use of an ICP monitor is significantly associated with improved survival in patients with severe head injury.

https://thejns.org/doi/abs/10.3171/2018.7.JNS18270

KEYWORDS traumatic brain injury; Glasgow Coma Scale; ICP monitoring; intracranial pressure

$\mathrm{T}$ HE incidence of traumatic brain injury (TBI) is increasing worldwide, and there is an urgent need for both preventative measures and better treatment. ${ }^{22}$ Clinical trials of new measures to reduce the extent of primary injury in TBI have so far been largely futile. ${ }^{16}$

It has been postulated that the treatment of patients with severe head injury is facilitated by intracranial pressure (ICP) monitoring. 5 The literature, however, contains conflicting reports. Some studies suggest no, or even detrimental, effects of using ICP monitors, while others indicate a positive effect of ICP monitoring on outcome. . $^{7,15,23}$ Common to these studies is the observational design and limited adjustment for variables known to be associated with a poor outcome. In 2012, Chesnut et al. published a randomized controlled trial that included patients with TBI from Bolivia and Ecuador. ${ }^{6}$ This trial reported a non-

ABBREVIATIONS AIS = Abbreviated Injury Scale; ASA = American Society of Anesthesiologists Physical Status Classification; BEST-TRIP = Benchmark Evidence from South American Trials: Treatment of Intracranial Pressure; BTF = Brain Trauma Foundation; $\mathrm{Cl}=$ confidence interval; $\mathrm{CPP}=$ cerebral perfusion pressure; EVD = external ventricular drain; GB = generalized boosting; GCS = Glasgow Coma Scale; ICP = intracranial pressure; ISS = Injury Severity Score; OR = odds ratio; OUH-U = Oslo University Hospital, Ullevål; TBI = traumatic brain injury.

SUBMITTED January 31, 2018. ACCEPTED July 24, 2018

INCLUDE WHEN CITING Published online December 21, 2018; DOI: 10.3171/2018.7.JNS18270. 
significant difference in outcome between an ICP-monitored group and a control group monitored by clinical examination and serial CT, suggesting that the use of ICP monitors is unnecessary in the treatment of TBI. Objections to the study include the external validity of the findings, that it was underpowered, and that the control arm in the study could reflect a trial effect. ${ }^{12}$ Therefore, it is still unclear whether patients with severe TBI benefit from ICP monitoring.

In this study we investigated the effect of ICP monitoring on 30-day survival. We tried to overcome some of the limitations of earlier retrospective studies by utilizing a propensity score-weighted analysis of data from a prospectively maintained registry at a level I trauma hospital, thus ensuring good face validity and a relevant clinical setting for our population.

\section{Methods \\ Study Population}

Oslo University Hospital, Ullevål (OUH-U) is the major trauma-care facility in the South-Eastern Norway Regional Health Authority. The neurosurgical department at OUH-U treats all severe neurotrauma within this region, covering a population of approximately 2.8 million people in a geographical area of $110,000 \mathrm{~km}^{2} .^{21}$

Jones et al. have previously described the trauma registry and trauma logistics at OUH-U. ${ }^{13}$ Briefly, trauma patients with severe injuries and patients with suspected isolated severe neurotrauma are transported and admitted directly to OUH-U from the entire health region. Patients with less severe injuries are admitted to other acute care hospitals but transferred to OUH-U later if necessary. The modes of transportation between the scene of an accident and referring hospitals and OUH-U are helicopters manned with anesthesiologists or ground ambulances manned with paramedics.

\section{Study Criteria}

All patients with Injury Severity Scores (ISSs) $\geq 10$ admitted to OUH-U within 24 hours of the trauma, patients with penetrating injuries to the torso, patients with injuries proximal to the knees and elbows, and all cases admitted by the trauma team are included in the trauma registry. ${ }^{3}$ Two registrars, both nurse anesthetists with trauma team experience and with formal education in injury classification according to the Abbreviated Injury Scale (AIS 90, update 98 ), prospectively update the registry. ${ }^{2}$ The treatment of patients with TBI at OUH-U has gradually improved during the study period, consistently conforming to the Brain Trauma Foundation (BTF) guidelines. ${ }^{5}$

Although the indications for inserting an ICP monitor have been rooted in the BTF guidelines, the ultimate decision was made at the discretion of the attending neurosurgeon. The changes in trauma team factors, neurosurgical presence and competence, and neurointensive care systems at OUH-U have recently been published by Søvik et al. ${ }^{25}$ Briefly, the goals of TBI treatment have been to maintain ICP at $<20 \mathrm{~mm} \mathrm{Hg}$ and the cerebral perfusion pressure (CPP) at $>60 \mathrm{~mm} \mathrm{Hg}$. A staircase-protocolled approach using sedation, osmolar therapy, normothermia,
CSF drainage, barbiturates, and hemicraniectomy was utilized to fulfill the ICP and CPP goals. ${ }^{26}$

In this study, our prospective registry was searched for the records of all patients admitted with either Glasgow Coma Scale (GCS) scores $<9$ or head AIS scores $\geq 2$ over 12 years (January 1, 2002, to December 31, 2013). We retrieved the records of 6936 patients. Risk factors for intracranial hypertension are either GCS scores 3-8 and an abnormal CT scan (here defined as head AIS score $\geq 3$ ), or GCS scores 3-8 with a normal CT scan, but with two or more of the following factors: age $>40$ years, motor posturing, or systolic blood pressure $<90 \mathrm{~mm} \mathrm{Hg} .{ }^{4}$ Hence, we filtered the 6936 patients according to these risk factors to define a population with a high risk of intracranial hypertension where it is most likely to detect a benefit of ICP monitoring. This filtering resulted in 1509 patients. Patients deemed unsalvageable were removed, by deleting those who died within 24 hours of admittance $(n=177)$. Patients with a maximum head AIS score equal to 6 were also discarded due to poor prognosis $(n=6)$. We retrieved information about the resulting patients on 53 different variables (the full list is available upon request). The most salient variables included information on demographics (age, sex), trauma (date, time, first hospital, ISS), physiological parameters on admission (blood pressure, oxygen saturation, heart rate, preinjury comorbidity scoring according to The American Society of Anesthesiologists Physical Status Classification [ASA] score), brain trauma (GCS score including component scores; maximum head AIS score; ICD-10 diagnoses; and operations according to the Nordic Medico-Statistical Committee classification of surgical procedures), and outcome (date of death, survival at 30 days [yes/no]).

In our department we have routinely utilized a parenchymal ICP probe (Codman Microsensor ICP transducer) in managing patients with TBI. External ventricular drains (EVDs) were inserted only if medical therapy failed to control ICP and/or ventriculomegaly was present on the CT scans. Thus, if an EVD was inserted, the drain was used for therapy, not monitoring, and the patient was monitored concurrently with a parenchymal ICP probe.

Before data extraction, all data elements were thoroughly screened for inconsistencies and nonlogical values, in compliance with the OUH-U trauma registry data validation protocol.

\section{Statistical Analysis}

First, the data set was described in two groups according to whether the patient had an ICP monitor inserted or not, using the Wilcoxon test for continuous data and the Pearson chi-square test for categorical data. A proportional odds model was used to check for differences in ordinal data.

There were missing data for the oxygen saturation, heart rate, and systolic blood pressure variables $(8.9 \%$, $4.9 \%$, and $4.3 \%$ of variables, respectively). Full information on all variables was present in 1186 patients (89.4\%). The pattern of missingness was evaluated and found to be reasonably missing at random. In order not to remove these patients from the analysis and consequently reduce the power of the analysis and possibly increase bias, we 
imputed a total of 10 data sets using multiple imputation..$^{28}$ The uncertainty of the imputed value is reflected by the range of imputed values over the 10 data sets and is accounted for in the final analysis.

Due to covariate imbalance between the two groups (with or without an ICP monitor), the analysis with regard to ICP monitoring could be biased. Consequently, we used different propensity score methods to determine if we could achieve balance in the distribution of covariates between the two groups. Briefly, the probability of receiving treatment is modeled based on the other covariates. This probability can be calculated from a logistic regression model or from machine-learning algorithms. We fitted both a logistic regression model and a generalized boosting (GB) model using the twang package in $\mathrm{R}$ with 15,000 iterations to define the probability of treatment. All variables in Table 1 were used to predict treatment, apart from the "dead within 30 days" variable.

This probability of treatment was subsequently used for matching. ${ }^{27}$ The following approaches were investigated for matching the propensity score derived from both logistic regression and the GB model: full matching, nearest neighbor matching, genetic matching, subclassification, and weighting. The GB method with a weighted analysis outperformed the other approaches using the metric of standardized differences in means between the two groups (data available upon request).

The final effect of ICP monitoring on survival was calculated using weighted uni- and multivariate regression on each of the imputed data sets. The aggregated coefficients from the 10 data sets were obtained by using a cluster approach detailed in the survey package and by using Rubin's rule adapted in the MItools package. The results from these different methods were compared and found to be similar. Furthermore, a weighted KaplanMeier curve is presented. For reference, uni- and multivariate logistic regression models were calculated using only the patients from the original sample with complete information.

Propensity-score weighting can primarily adjust for observed variables. Unobserved variables are only accounted for to the extent that they are correlated with the observed variables. ${ }^{27} \mathrm{~A}$ sensitivity analysis can indicate the amount of hidden bias from unobserved variables necessary to invalidate the findings from the propensity-score weighted analysis. We performed a sensitivity analysis for binary responses in each of the imputed data sets according to Rosenbaum's method. The genetic matching procedure performed marginally poorer than the weighted matching method, but was used due to the convenience of the binarysens function in the Rbounds package.

The statistical program $\mathrm{R}$ was used for all analyses (version 3.2; http://www.R-project.org). A p value $<0.05$ was considered statistically significant.

\section{Ethics}

The Oslo University Hospital Data Protection Officer, in this matter representing the Regional Committee for Medical and Health Research Ethics and the Norwegian Data Protection Authority, considered the study exempt from patient consent requirements.
TABLE 1. Patient characteristics

\begin{tabular}{|c|c|c|c|c|}
\hline \multirow[b]{2}{*}{ Variable } & \multirow{2}{*}{$\begin{array}{c}\text { No. of } \\
\text { Patients }\end{array}$} & \multicolumn{2}{|c|}{ ICP Monitor } & \multirow{2}{*}{$\begin{array}{c}p \\
\text { Value }\end{array}$} \\
\hline & & No $(n=570)$ & Yes $(n=757)$ & \\
\hline GCS score & 1327 & & & 0.247 \\
\hline 3 & & $44.1 \%(164)$ & $55.9 \%(208)$ & \\
\hline 4 & & $30.4 \%(41)$ & $69.6 \%(94)$ & \\
\hline 5 & & $40.3 \%(71)$ & $59.7 \%(105)$ & \\
\hline 6 & & $45.7 \%(107)$ & $54.3 \%$ (127) & \\
\hline 7 & & $46.0 \%(87)$ & $54.0 \%(102)$ & \\
\hline 8 & & $45.2 \%(100)$ & $54.8 \%(121)$ & \\
\hline Admission & 1327 & & & 0.554 \\
\hline Primary & & $43.6 \%(348)$ & $56.4 \%(450)$ & \\
\hline Secondary & & $42.0 \%(222)$ & $58.0 \%$ (307) & \\
\hline Age $(y r s)^{*}$ & 1325 & $22.2 / 41.4 / 64.2$ & $23.4 / 38.1 / 56.6$ & 0.014 \\
\hline Sex & 1327 & & & 0.146 \\
\hline Female & & $46.3 \%(161)$ & $53.7 \%(187)$ & \\
\hline Male & & $41.8 \%(409)$ & $58.2 \%(570)$ & \\
\hline Year & 1327 & & & $<0.001$ \\
\hline 2002 & & $72.2 \%(83)$ & $27.8 \%(32)$ & \\
\hline 2003 & & $71.7 \%(66)$ & $28.3 \%(26)$ & \\
\hline 2004 & & $51.4 \%(57)$ & $48.6 \%(54)$ & \\
\hline 2005 & & $45.4 \%(59)$ & $54.6 \%(71)$ & \\
\hline 2006 & & $39.8 \%(45)$ & $60.2 \%(68)$ & \\
\hline 2007 & & $39.4 \%(56)$ & $60.6 \%(86)$ & \\
\hline 2008 & & $31.4 \%(33)$ & $68.6 \%(72)$ & \\
\hline 2009 & & $32.1 \%(35)$ & $67.9 \%(74)$ & \\
\hline 2010 & & $41.2 \%(33)$ & $58.8 \%(47)$ & \\
\hline 2011 & & $33.0 \%(38)$ & $67.0 \%(77)$ & \\
\hline 2012 & & $30.2 \%(32)$ & $69.8 \%(74)$ & \\
\hline 2013 & & $30.3 \%(33)$ & $69.7 \%(76)$ & \\
\hline $\begin{array}{l}\text { Epidural hema- } \\
\text { toma }\end{array}$ & 1327 & & & $<0.001$ \\
\hline No & & $44.3 \%(544)$ & $55.7 \%(685)$ & \\
\hline Yes & & $26.5 \%(26)$ & $73.5 \%(72)$ & \\
\hline $\begin{array}{l}\text { Subdural } \\
\text { hematoma }\end{array}$ & 1327 & & & $<0.001$ \\
\hline No & & $47.1 \%(520)$ & $52.9 \%(585)$ & \\
\hline Yes & & $22.5 \%(50)$ & $77.5 \%(172)$ & \\
\hline Contusion & 1327 & & & $<0.001$ \\
\hline No & & $44.5 \%$ (554) & $55.5 \%$ (692) & \\
\hline Yes & & $19.8 \%(16)$ & $80.2 \%(65)$ & \\
\hline $\begin{array}{l}\text { Penetrating } \\
\text { injury }\end{array}$ & 1327 & & & 0.313 \\
\hline No & & $43.1 \%(564)$ & $56.9 \%(744)$ & \\
\hline Yes & & $31.6 \%(6)$ & $68.4 \%(13)$ & \\
\hline Cranial fracture & 1327 & & & $<0.001$ \\
\hline No & & $44.7 \%(552)$ & $55.3 \%$ (684) & \\
\hline Yes & & $19.8 \%(18)$ & $80.2 \%(73)$ & \\
\hline
\end{tabular}

CONTINUED ON PAGE $1899 »$ 
» CONTINUED FROM PAGE 1898

TABLE 1. Patient characteristics

\begin{tabular}{|c|c|c|c|c|}
\hline \multirow[b]{2}{*}{ Variable } & \multirow{2}{*}{$\begin{array}{c}\text { No. of } \\
\text { Patients }\end{array}$} & \multicolumn{2}{|c|}{ ICP Monitor } & \multirow{2}{*}{$\begin{array}{c}p \\
\text { Value }\end{array}$} \\
\hline & & No $(n=570)$ & Yes $(n=757)$ & \\
\hline $\begin{array}{l}\text { Max head AIS } \\
\text { score }\end{array}$ & 1327 & & & $<0.001$ \\
\hline $2 \& 3$ & & $66.3 \%(185)$ & $33.7 \%(94)$ & \\
\hline 4 & & $36.9 \%(117)$ & $63.1 \%(200)$ & \\
\hline 5 & & $36.7 \%(268)$ & $63.3 \%(463)$ & \\
\hline ISS (points) ${ }^{*}$ & 1327 & $17 / 26 / 34$ & $26 / 29 / 38$ & $<0.001$ \\
\hline ASA & 1320 & & & 0.032 \\
\hline 1 & & $41.2 \%(333)$ & $58.8 \%(475)$ & \\
\hline 2 & & $40.4 \%(112)$ & $59.6 \%(165)$ & \\
\hline 3 & & $50.5 \%(104)$ & $49.5 \%(102)$ & \\
\hline 4 & & $55.2 \%(16)$ & $44.8 \%(13)$ & \\
\hline $\begin{array}{c}\text { Systolic blood } \\
\text { pressure } \\
(\mathrm{mm} \mathrm{Hg})^{*}\end{array}$ & 1269 & $105 / 130 / 150$ & $105 / 122 / 140$ & 0.054 \\
\hline $\begin{array}{l}\text { Oxygen satura- } \\
\text { tion }(\%)^{*}\end{array}$ & 1208 & $97 / 99 / 100$ & $97 / 99 / 100$ & 0.066 \\
\hline $\begin{array}{l}\text { Heart rate } \\
\qquad \text { (beats } / \mathrm{min})^{*}\end{array}$ & 1262 & 72.0/85.0/105.0 & 71.0/85.0/103.5 & 0.690 \\
\hline $\begin{array}{l}\text { Dead within } 30 \\
\text { days }\end{array}$ & 1327 & & & $<0.001$ \\
\hline No & & $36.8 \%(370)$ & $63.2 \%(635)$ & \\
\hline Yes & & $62.1 \%(200)$ & $37.9 \%(122)$ & \\
\hline
\end{tabular}

${ }^{*}$ Data given as lower quartile/median/upper quartile.

\section{Results}

We retrieved the records of 1327 patients fulfilling the inclusion criteria of having sustained a severe TBI and surviving $\geq 24$ hours. The overall mortality rate in our cohort was $24.3 \%$ (322 patients). Patients in the ICP-monitored group tended to be younger $(\mathrm{p}=0.01)$. Furthermore, there was a clear tendency for increasing use of ICP monitors in the time period from 2002 to 2013 ( $\mathrm{p}<0.001$ ). Patients with more severe anatomical head injuries were also more likely to have an ICP monitor inserted $(p<0.001)$. Further information about the study population is provided in Table 1.

The weights from the GB model were calculated and the balance between the ICP-monitored and non-ICPmonitored group was investigated and found to be within 0.2 standardized means of each other in all 10 imputed data sets (Fig. 1 left). Furthermore, the relative influences of the different variables in predicting whether an ICP monitor was inserted or not in the GB models were calculated (Fig. 1 right). Age, year, ISS sum, systolic blood pressure on admission, maximum AIS head score, and heart rate accounted for $86 \%$ of the cumulative influence in predicting whether the patient received an ICP monitor or not.

The GB-weighted Kaplan-Meier curve for the aggregated 10 imputed data sets demonstrated improved survival in patients who had an ICP monitor inserted (log-rank test, $\mathrm{p}<0.001$ ). The 30 -day mortality rate was $35.1 \%$ (200 patients, $95 \%$ confidence interval [CI] $31.3 \%-39.1 \%$ ) in the group without ICP monitors and $16.1 \%$ (122 patients, 95\% CI 13.6\%-18.9\%) in the group with ICP monitors (Fig. 2).

The impact of ICP monitors on 30-day mortality was found to be beneficial for the adjusted analyses in both the complete case data set (odds ratio [OR] 0.23, 95\% CI $0.16-0.33)$ and the aggregated, multiple imputed, propensity-weighted data set (OR $0.22,95 \%$ CI $0.15-0.35$; both $\mathrm{p}<0.001)$. Moreover, decreasing maximum AIS head scores were associated with reduced 30-day mortality compared to maximum AIS head scores of 5 (all $\mathrm{p}<$ 0.001). Likewise, decreasing ASA scores were associated with reduced odds of 30-day mortality, compared to an ASA score of 4 (all $\mathrm{p}<0.05$ ). Epidural hematomas and increasing oxygen saturation were also associated with reduced odds of 30 -day mortality $(\mathrm{p}<0.01)$. Increasing age was the only factor, identified in both the complete case analysis and in the imputed data set with propensity-score weighted analysis, found to be associated with increased odds of 30-day mortality (all $\mathrm{p}<0.001$ ). For further details and the coefficients, see Fig. 3.

The sensitivity analysis for the different imputed propensity-weighted models $(\mathrm{n}=10)$ demonstrated that a median gamma of 2.41 (range 2.08-2.69) is needed to invalidate the beneficial effect of inserting an ICP monitor. This implies that to attribute the improved survival rate in the group with an ICP monitor due to an unobserved covariate, rather than to the ICP monitor, the unobserved covariate would need to account for a 2.41 increase in the odds of receiving an ICP monitor.

\section{Discussion}

The results from our study indicate a clear survival benefit of using ICP monitors in patients with severe brain injury who survive the first 24 hours after injury. The adjusted ORs for 30-day mortality in both the complete case analysis and in the imputed data set with propensity scoreweighted analysis were 0.23 (95\% CI 0.16-0.33) and 0.22 (95\% CI $0.15-0.35$ ), respectively (both $\mathrm{p}<0.001$ ).

This is in contrast to the randomized controlled BESTTRIP (Benchmark Evidence from South American Trials: Treatment of Intracranial Pressure) trial, in which the effect of ICP monitoring was insignificant. ${ }^{6}$ The BESTTRIP trial was designed to compare the effect of ICP monitoring to best clinical monitoring. Best clinical monitoring entailed looking for signs indicating increased ICP clinically or radiologically. Hence, BEST-TRIP was a trial comparing ICP read by an ICP monitor to clinical and radiological proxy signs of increased ICP. Thus, the concept of ICP in the treatment of severe TBI was not investigated. In the event of clinical or radiological signs of increased ICP, adjunctive ICP-lowering treatment measures were started. Such treatment measures were utilized to a larger extent in the clinically monitored group underscoring the implicit focus on ICP, even in the non-ICP-monitored group.

The clinical examination, monitoring, and documentation of the patients were probably more comprehensive than standard clinical practice. Consequently, by providing more vigilant clinical monitoring, the non-ICP-moni- 


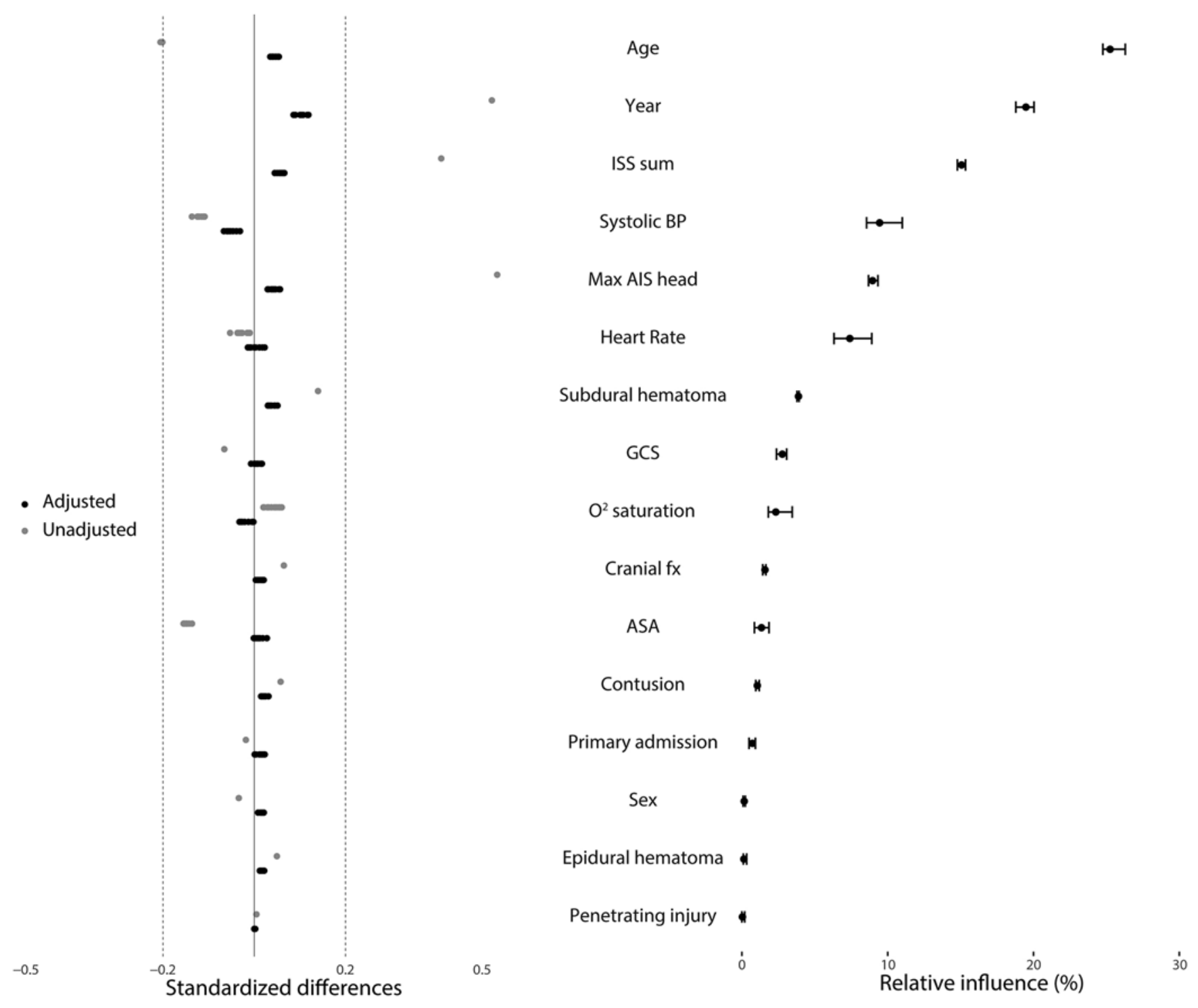

FIG. 1. The left pane/ demonstrates the effect of weighting the propensity score obtained from the GB model on standardized differences between the ICP-monitored and -nonmonitored groups. The different points indicate the different results from the 10 imputed data sets. The right panel indicates the relative influence of the variable on the final multivariate model from the GB model. $\mathrm{BP}=$ blood pressure; $f x=$ fracture.

tored arm in the BEST-TRIP trial could detect more detrimental episodes of increased ICP than were detected in the non-ICP-monitored group in our cohort. Thus, the BEST-TRIP trial could have reduced the relative impact of the ICP monitor on outcome by providing excellent clinical monitoring.

Cremer et al. compared outcomes between two centers in Holland, in which one of the centers subscribed to the tenets of the BTF guidelines (211 patients) while the other center used clinical monitoring in the treatment of severe TBI (122 patients). ${ }^{7}$ These investigators failed to detect a significant difference with respect to outcome between these centers, thus agreeing with the results from BESTTRIP and maintaining the external validity for industrialized countries. Likewise, Shafi et al. analyzed the National
Trauma Data Bank run by The American College of Surgeons in the time period from 1994 to $2001 .{ }^{23}$ Of almost 500,000 trauma victims, they identified 1646 meeting the BTF criteria for ICP monitoring. Only $43 \%$ received an ICP monitor (708 patients). In the ICP-monitored group they found a $45 \%$ reduction in survival (OR $0.55,95 \%$ CI $0.39-0.76, \mathrm{p}<0.001)$. Head AIS score and the use of craniotomies were significantly higher in the ICP-monitored group, and GCS score at the scene of the accident was significantly lower. Although they used multivariate techniques that to a certain extent can adjust for group differences, we believe their findings also could be due to confounding by indication.

Our results are compatible with findings in other observational studies, indicating that ICP monitoring is benefi- 


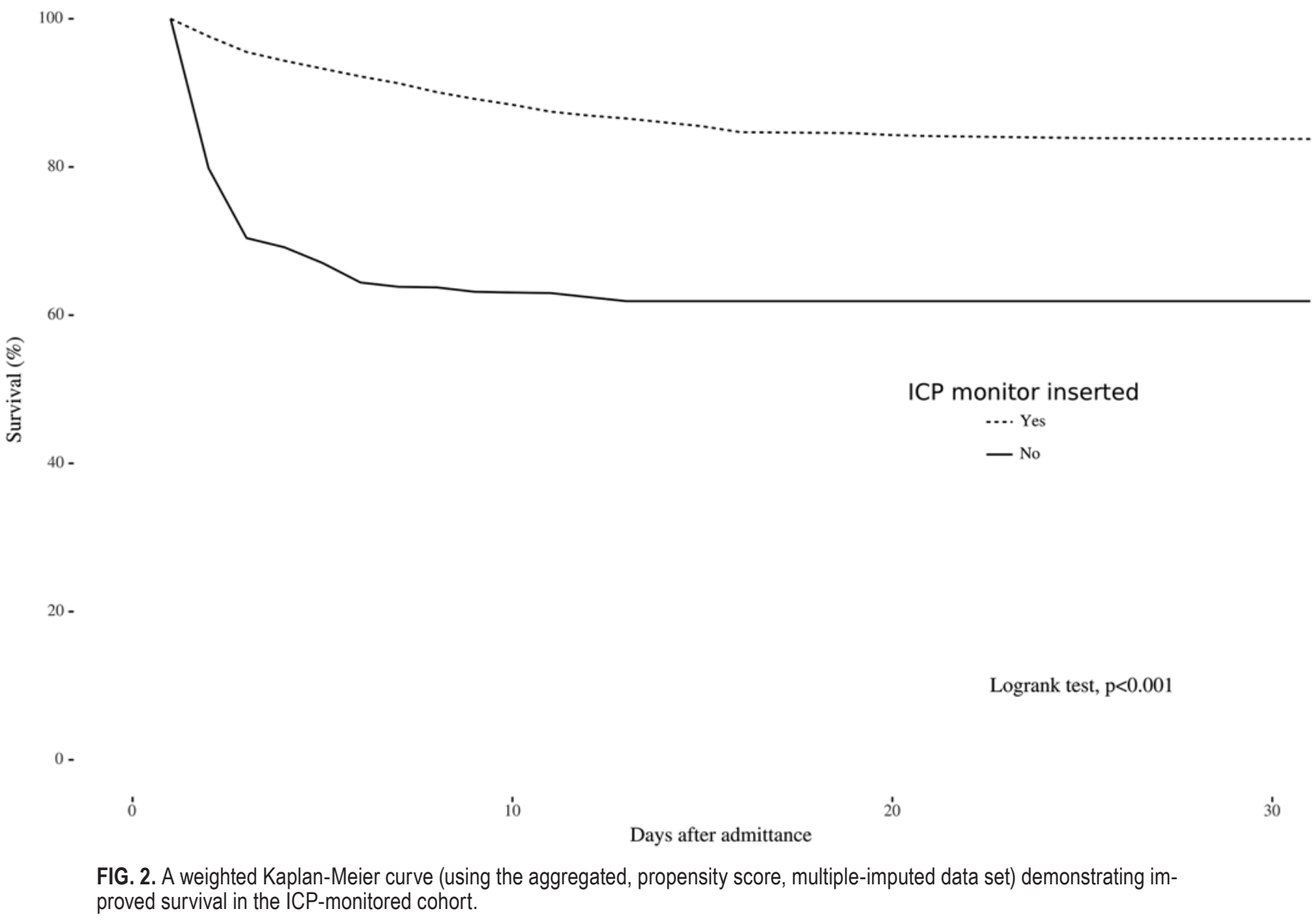

cial for patients with severe TBI fulfilling the BTF criteria for ICP monitoring. ${ }^{1,8,14,17,30,31}$ Moreover, in a recent metaanalysis of both observational and randomized controlled trials, the use of ICP monitors was found to be beneficial. ${ }^{24}$

The heterogeneity in the effect of ICP monitors on outcome in the observational studies is perplexing. However, the definition of a futile prognosis is different among the studies. A study that includes all patients would also include patients defined to be unsalvageable. Unsalvageable patients would not receive an ICP monitor, be assigned to the non-ICP-monitored group, and most likely die. This practice would inflate the ratio of dead patients in the nonICP-monitored group. Conversely, by defining futility as death several days after admittance, the relative impact of ICP monitoring will be reduced. The findings by Shafi et al. could be consistent with this, as they used a futility cutoff at 48 hours. ${ }^{23}$ However, this explanation is contested by the findings of Cremer et al. and Griesdale et al. who both failed to identify a significant effect of ICP monitoring and used futility cutoffs at 24 and 12 hours, respectively. ${ }^{7,10}$

\section{Strengths and Limitations}

A major strength of our study is that it includes 1327 patients meeting the 2007 BTF level II and III criteria for being at high risk of intracranial hypertension. ${ }^{4}$ Thus, by restricting the study population to the population most likely to develop intracranial hypertension, it can be argued that we increase the power of the study.

Likewise, imputation of the missing variables instead of only using a complete case analysis ensures statistical power by not decreasing the effective sample size. The imputation technique also obviates any bias that could have been introduced by a complete case analysis if the missing cases were not missing completely at random. ${ }^{11}$

In randomized controlled trials, the randomization process conceptually guarantees that all confounders, both observed and nonobserved, will be evenly assigned between the groups. This process thus ensures that the groups only differ with respect to the intervention, allowing causal inferences to be made. Our study consists of an observational cohort stratified by an intervention. The ICP-monitored and -nonmonitored groups are not assumed to be equal with regard to the distribution of confounding factors. Consequently, a causal association between ICP monitoring and survival is difficult to defend. However, we have used a weighted analysis approach based on a propensity score derived from a GB model demonstrating similar covariate distribution between the two groups. This methodology can only account for the observed confounders. However, we argue that many of the observed confounders used in our model are well-established outcome predic- 


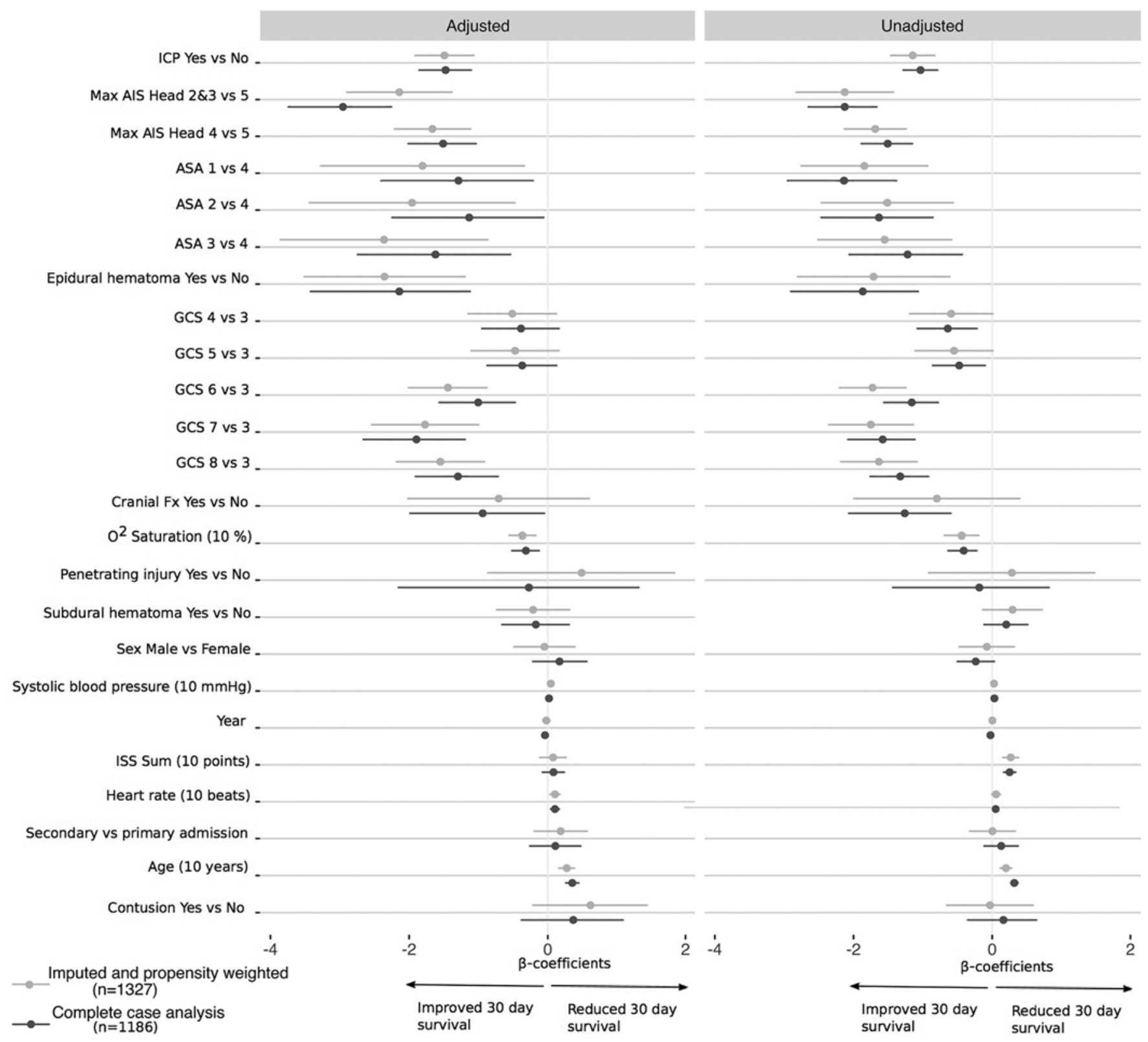

FIG. 3. Coefficient plot. The unadjusted and adjusted $\beta$-coefficients and their associated $95 \% \mathrm{Cls}$ from the complete case data set using logistic regression are contrasted with the $\beta$-coefficients from the imputed, propensity score-weighted logistic regression analysis. The effect of ICP monitor insertion is significant for the adjusted analyses in both data sets $(\mathrm{OR} 0.23,95 \% \mathrm{Cl} 0.16-0.33$, and OR $0.22,95 \% \mathrm{Cl} 0.15-0.35$; both $\mathrm{p}<0.001$ ).

tors. ${ }^{20}$ Moreover, these well-established prognostic factors are very likely correlated with other unobserved outcome predictors. This correlation mitigates the effect of any unobserved outcome predictors. ${ }^{27}$ The sensitivity analysis indicates that an unobserved uncorrelated predictor would have to be differentially assigned between the two groups with an OR of 2.41 before the effect of ICP monitoring is insignificant. Finding such a strong uncorrelated outcome predictor is improbable, given that the strongest multivariate predictor in the IMPACT (International Mission for Prognosis and Analysis of Clinical Trials in TBI) study had an OR of 2.23 for CT class (Model A). ${ }^{20}$ Consequently, we argue for a causal significant effect of ICP monitoring on outcome despite the shortcomings of an observational cohort.

The propensity score was obtained using both the fitted probabilities from a logistic regression model and from a GB algorithm. The latter technique automatically incorporates the effect of nonlinearity and interactions..$^{19}$ These theoretical advantages could improve the model fit in a GB model and contribute to the almost equal distribution of the salient prognostic factors in TBI between the groups with and without an ICP monitor noted in Fig. 1. This distribution of covariates supports the notion that quasi-cau- 
sality can be inferred. Furthermore, imputation techniques are often recommended to reduce bias and increase statistical power rather than just analyzing the complete cases. ${ }^{11}$

A major caveat to appreciate the effect of all types of monitoring on outcome is that the monitor itself cannot be assumed to influence the outcome. Rather, it is the decisions and treatment based on the output from the monitor that is presumed to affect outcome. In this study, as in most other observational studies investigating the effect of ICP monitoring, we have taken the approach that just inserting an ICP monitor could have an impact on outcome. This is a simplistic approach ignoring the extent to which the ICP and CPP values have actually been adequately treated. In contrast, requiring compliance with either the ICP and/or CPP protocol recommendations will effectively make the problem unamenable to scientific scrutiny if the outcome is mortality rate, because a moribund patient with TBI will be in violation of protocols. To circumvent this issue, other studies have reported the proportion of time spent above a certain threshold, amount of ICP-lowering measures used in treatment, and area under the ICP curve. ${ }^{6,18,29}$ However, the effects of the ICP-lowering measures could act through other mechanisms than lowering ICP alone, thus complicating the prospect of establishing causality between ICP and outcome.

\section{Conclusions}

ICP monitoring was associated with improved survival in our cohort of patients with severe TBI, even after controlling for baseline severity of the head injury using a propensity score-weighted approach. Our results support the continued use of ICP monitors in patients with severe TBI.

\section{References}

1. Alali AS, Fowler RA, Mainprize TG, Scales DC, Kiss A, de Mestral C, et al: Intracranial pressure monitoring in severe traumatic brain injury: results from the American College of Surgeons Trauma Quality Improvement Program. J Neurotrauma 30:1737-1746, 2013

2. Association for the Advancement of Automotive Medicine: The Abbreviated Injury Scale 1990 Revision: Update 98. Barrington, IL: Association for the Advancement of Automotive Medicine, 1998

3. Baker SP, O'Neill B, Haddon W Jr, Long WB: The injury severity score: a method for describing patients with multiple injuries and evaluating emergency care. J Trauma 14:187196, 1974

4. Brain Trauma Foundation: Guidelines for the management of severe traumatic brain injury. J Neurotrauma 24 (Suppl 1):S1-S106, 2007

5. Carney N, Totten AM, O'Reilly C, Ullman JS, Hawryluk GW, Bell MJ, et al: Guidelines for the Management of Severe Traumatic Brain Injury, Fourth Edition. Neurosurgery 80:6-15, 2017

6. Chesnut RM, Temkin N, Carney N, Dikmen S, Rondina C, Videtta W, et al: A trial of intracranial-pressure monitoring in traumatic brain injury. N Engl J Med 367:2471-2481, 2012

7. Cremer OL, van Dijk GW, van Wensen E, Brekelmans GJ, Moons KG, Leenen LP, et al: Effect of intracranial pressure monitoring and targeted intensive care on functional outcome after severe head injury. Crit Care Med 33:2207-2213, 2005
8. Dawes AJ, Sacks GD, Cryer HG, Gruen JP, Preston C, Gorospe D, et al: Intracranial pressure monitoring and inpatient mortality in severe traumatic brain injury: a propensity score-matched analysis. J Trauma Acute Care Surg 78:492-502, 2015

9. Fakhry SM, Trask AL, Waller MA, Watts DD: Management of brain-injured patients by an evidence-based medicine protocol improves outcomes and decreases hospital charges. J Trauma 56:492-500, 2004

10. Griesdale DE, McEwen J, Kurth T, Chittock DR: External ventricular drains and mortality in patients with severe traumatic brain injury. Can J Neurol Sci 37:43-48, 2010

11. Harrell JFE: Regression Modeling Strategies: With Applications to Linear Models, Logistic and Ordinal Regression, and Survival Analysis. Cham, Switzerland: Springer International Publishing, 2015

12. Hutchinson PJ, Kolias AG, Czosnyka M, Kirkpatrick PJ, Pickard JD, Menon DK: Intracranial pressure monitoring in severe traumatic brain injury. BMJ 346:f1000, 2013

13. Jones JM, Skaga NO, Søvik S, Lossius HM, Eken T: Norwegian survival prediction model in trauma: modelling effects of anatomic injury, acute physiology, age, and co-morbidity. Acta Anaesthesiol Scand 58:303-315, 2014

14. Kostić A, Stefanović I, Novak V, Veselinović D, Ivanov G, Veselinović A: Prognostic significance of intracranial pressure monitoring and intracranial hypertension in severe brain trauma patients. Med Pregl 64:461-465, 2011

15. Lane PL, Skoretz TG, Doig G, Girotti MJ: Intracranial pressure monitoring and outcomes after traumatic brain injury. Can J Surg 43:442-448, 2000

16. Maas AIR, Roozenbeek B, Manley GT: Clinical trials in traumatic brain injury: past experience and current developments. Neurotherapeutics 7:115-126, 2010

17. MacLaughlin BW, Plurad DS, Sheppard W, Bricker S, Bongard F, Neville A, et al: The impact of intracranial pressure monitoring on mortality after severe traumatic brain injury. Am J Surg 210:1082-1087, 2015

18. Marmarou A, Anderson RL, Ward JD, Choi SC, Young HF, Eisenberg HM, et al: Impact of ICP instability and hypotension on outcome in patients with severe head trauma. J Neurosurg 75 Suppl:S59-S66, 1991

19. McCaffrey DF, Ridgeway G, Morral AR: Propensity score estimation with boosted regression for evaluating causal effects in observational studies. Psychol Methods 9:403-425, 2004

20. Murray GD, Butcher I, McHugh GS, Lu J, Mushkudiani NA, Maas AIR, et al: Multivariable prognostic analysis in traumatic brain injury: results from the IMPACT study. J Neurotrauma 24:329-337, 2007

21. Rønning P, Gunstad PO, Skaga NO, Langmoen IA, Stavem $\mathrm{K}$, Helseth E: The impact of blood ethanol concentration on the classification of head injury severity in traumatic brain injury. Brain Inj 29:1648-1653, 2015

22. Roozenbeek B, Maas AI, Menon DK: Changing patterns in the epidemiology of traumatic brain injury. Nat Rev Neurol 9:231-236, 2013

23. Shafi S, Diaz-Arrastia R, Madden C, Gentilello L: Intracranial pressure monitoring in brain-injured patients is associated with worsening of survival. J Trauma 64:335-340, 2008

24. Shen L, Wang Z, Su Z, Qiu S, Xu J, Zhou Y, et al: Effects of intracranial pressure monitoring on mortality in patients with severe traumatic brain injury: a meta-analysis. PLoS One 11:e0168901, 2016

25. Søvik S, Skaga NO, Hanoa R, Eken T: Sudden survival improvement in critical neurotrauma: an exploratory analysis using a stratified statistical process control technique. Injury 45:1722-1730, 2014

26. Stocchetti N, Maas AIR: Traumatic intracranial hypertension. N Engl J Med 370:2121-2130, 2014 
27. Stuart EA: Matching methods for causal inference: A review and a look forward. Stat Sci 25:1-21, 2010

28. van Buuren S, Groothuis-Oudshoorn K: mice: Multivariate Imputation by Chained Equations in R. J Stat Softw 45:167, 2011

29. Vik A, Nag T, Fredriksli OA, Skandsen T, Moen KG, Schirmer-Mikalsen K, et al: Relationship of "dose" of intracranial hypertension to outcome in severe traumatic brain injury. J Neurosurg 109:678-684, 2008

30. You W, Feng J, Tang Q, Cao J, Wang L, Lei J, et al: Intraventricular intracranial pressure monitoring improves the outcome of older adults with severe traumatic brain injury: an observational, prospective study. BMC Anesthesiol 16:35, 2016

31. Yuan Q, Wu X, Cheng H, Yang C, Wang Y, Wang E, et al: Is intracranial pressure monitoring of patients with diffuse traumatic brain injury valuable? An observational multicenter study. Neurosurgery 78:361-369, 2016

\section{Disclosures}

The authors report no conflict of interest concerning the materials or methods used in this study or the findings specified in this paper.

\section{Author Contributions}

Conception and design: Rønning, Langmoen. Acquisition of data: Skaga. Analysis and interpretation of data: Rønning, Stavem. Drafting the article: Rønning. Critically revising the article: all authors. Reviewed submitted version of manuscript: Rønning, Helseth, Skaga, Stavem. Approved the final version of the manuscript on behalf of all authors: Rønning. Statistical analysis: Rønning, Stavem. Administrative/technical/material support: Helseth, Langmoen. Study supervision: Langmoen.

\section{Correspondence}

Pål Rønning: Oslo University Hospital, Oslo, Norway. palronning@gmail.com. 